HEINRICH AG; FERRAZ RM; RAGASSI CF; REIFSCHNEIDER FJB. 2015. Caracterização e avaliação de progênies autofecundadas de pimenta biquinho salmão. Horticultura Brasileira 33: 465-470. http://dx.doi.org/10.1590/S0102-053620150000400010

\title{
Caracterização e avaliação de progênies autofecundadas de pimenta biquinho salmão
}

\author{
Ana G Heinrich ${ }^{1}$; Rodrigo M Ferraz ${ }^{1}$; Carlos F Ragassi²; Francisco JB Reifschneider ${ }^{2}$ \\ 'Universidade de Brasília (UnB), Brasília-DF, Brasil; aninha_glaucia@hotmail.com; ferraz.rodrigo89@gmail.com; 2Embrapa Hortaliças, \\ Brasília-DF, Brasil; carlos.ragassi@embrapa.br; francisco.reifschneider@embrapa.br
}

\begin{abstract}
RESUMO
A pimenta biquinho vem sendo cada vez mais valorizada no mercado consumidor, especialmente por seu uso em forma de conservas, sendo atrativa principalmente pelo sabor suave e ausência de pungência. Apesar da grande demanda do mercado, são poucas as cultivares disponíveis e programas de melhoramento devem ser estabelecidos a fim de obter e liberar genótipos com características comerciais de interesse dos produtores e consumidores. Este trabalho relata os esforços de melhoramento feitos em uma população de biquinho vermelha segregante que combinaram seleção massal estratificada e pedigree (duas autofecundações), resultando na obtenção de 17 progênies. Estas foram caracterizadas considerando 11 descritores morfológicos e avaliadas quanto à produção de frutos em condições de campo. Adicionalmente, o teor de capsaicina foi determinado utilizando-se High Pressure Liquid Chromatography (HPLC). Com base nos resultados obtidos, foram selecionadas três progênies: a progênie CNPH 35.103 foi considerada a mais promissora, em termos de produção por planta $(1,40 \mathrm{~kg} /$ planta), além de apresentar forte aroma e parede do fruto mais espessa quando comparada às demais; CNPH 35.094 e 35.099 destacaram-se entre as demais devido à sua maior produção e aos teores de capsaicina próximos a zero, constituindo-se em genótipos com alto potencial para dar origem a uma cultivar de pimenta biquinho salmão.
\end{abstract}

Palavras-chave: Capsicum chinense, seleção, pimenta doce, coloração de frutos, pungência.

\begin{abstract}
Characterization and evaluation of salmon-colored biquinhotype pepper progenies

Capsicum chinense peppers are mostly native to Brazil and the biquinho (beaked)-type $C$. chinense pepper, aromatic and nonpungent, has been increasing in importance in the Brazilian fresh and pickled pepper market. Despite the increasing demand, a few cultivars are available to growers and breeding programs are required in order to offer interesting cultivars to producers and consumers. This paper reports breeding efforts made on a segregating red-fruited biquinho population which combined stratified mass selection and selfing for two generations before obtaining 17 inbred lines. The 17 progenies were characterized considering 11 morphological traits, and evaluated for fruit production under field conditions. Additionally, capsaicin content was determined using High Pressure Liquid Chromatography (HPLC). Based on the results, three progenies were selected and considered to have high potential: CNPH 35.103 was considered promising for future release as a cultivar due to high yield $(1.4 \mathrm{~kg} /$ plant), strong aroma and thick walls; and CNPH 35.094 and CNPH 35.099 were also high yielders and presented capsaicin content close to zero.
\end{abstract}

Keywords: Capsicum chinense, selection, sweet pepper, fruit color, pungency.

(Recebido para publicação em 27 de novembro de 2014; aceito em 7 de maio de 2015) (Received on November 27, 2014; accepted on May 7, 2015)

\begin{abstract}
A s pimentas e pimentões do gênero Capsicum, originários das Américas, representam parte valiosa da biodiversidade brasileira além de possuírem grande valor comercial (Ribeiro et al., 2008). Produtos de pimenta vermelha, pungentes e não pungentes, representam, em volume, uma das mais importantes commodities de tempero no mundo. Esses produtos adicionam aroma de especiarias e coloração aos alimentos, além de fornecerem vitaminas
\end{abstract}

A, $\mathrm{C}$ e $\mathrm{E}$ e minerais essenciais (Bosland \& Votava, 2000; Materska \& Perucka, 2005). Em 2010, foram cultivados cerca de 3,8 milhões ha de pimentas e pimentões por todo o mundo, totalizando uma produção de 30,6 milhões de toneladas (FAO, 2012).

O gênero Capsicum se caracteriza por uma grande diversidade genética e possui 30 espécies identificadas (DeWitt \& Bosland, 2009). Entretanto, apenas as espécies, C. annuum, C. baccatum,
C. chinense, $C$. frutescens e $C$. pubescens são consideradas domesticadas (Carvalho et al., 2003). Esse gênero engloba uma multiplicidade de formatos, cores e tamanhos de frutos que podem apresentar diferentes graus de pungência, determinada pela concentração de capsaicinoides, exclusivos desse gênero (Moscone et al., 2007; DeWitt \& Bosland, 2009). Mais de dez capsaicinoides já foram identificados; a capsaicina e a dihidrocapsaicina são as 
mais importantes, sendo que a primeira corresponde a cerca de $70 \%$ do total na maioria das variedades avaliadas por Kosuge \& Furuta (1970).

Capsicum chinense, diferente do que o nome sugere, é originária do hemisfério Ocidental, assim como todas as outras espécies de Capsicum. Há poucos trabalhos sobre a grande variedade de pimentas dessa espécie (Jarret \& Berke, 2008; Moses \& Umaharan, 2012; Faria et al., 2013), que é popular em todas as regiões tropicais e que tem na Bacia Amazônica a sua maior diversidade. Os frutos de $C$. chinense são caracterizados por uma grande diversidade, em termos de cores e podem ser suaves, doces e aromáticos, ou até mesmo extremamente pungentes (Ribeiro et al., 2008; Moura et al., 2010; Alvares et al., 2012). A cultivar Bhut Jolokia, representante dessa espécie e originária da Índia, é um híbrido interespecífico que ocorre naturalmente e é dita como uma das pimentas mais pungentes do mundo, com mais de 1.000.000 de Unidades de Calor Scoville (SHUs) (Bosland \& Baral, 2007; DeWitt \& Bosland, 2009). Os frutos de $C$. chinense apresentam forte aroma que é considerado muito distinto daquele das demais espécies de pimenta domesticada (Garruti et al., 2013). Entre as pimentas dessa espécie, habanero, pimenta-de-cheiro, murupi, biquinho, bode e cumari-do-pará são os tipos mais conhecidos (Ribeiro et al., 2008).

No Brasil, a espécie $C$. chinense é cultivada principalmente na região do triângulo mineiro, no estado de Minas Gerais (Carvalho et al., 2008). Grande parte dos pequenos agricultores brasileiros cultiva variedades locais de pimentas do gênero Capsicum que são obtidas a partir de vários ciclos de seleção realizados por eles, muitas vezes motivados pela falta de cultivares com características comerciais uniformes, devidamente registradas no Registro Nacional de Cultivares (RNC), do Ministério da Agricultura Pecuária e Abastecimento, e disponíveis no mercado.

Existem variações de consumo das pimentas em diferentes regiões do País e a pimenta biquinho pode atingir alto valor comercial, sendo uma alternativa interessante para os pequenos produtores. Os frutos do tipo biquinho são bastante apreciados, principalmente na forma processada como conserva, em função do aroma, da crocância e da ausência de pungência.

É possível desenvolver novos genótipos comerciais deste tipo de pimenta que tenham características estáveis e uniformes, doces e com cores distintas a partir da seleção de plantas em populações com variabilidade e que possam atender à crescente demanda por novas cultivares, ocupando assim, diferentes nichos de mercado (Carvalho et al., 2009). O sucesso no desenvolvimento de novas cultivares está diretamente associado à variabilidade genética dentro da espécie. O uso dessa variabilidade pelos melhoristas é o que permite, por meio de combinações genéticas, o surgimento de novos indivíduos mais adaptados, produtivos e resistentes a doenças, entre outras características (Souza et al., 2011).

No presente trabalho descrevem-se a seleção, a caracterização e a avaliação de progênies promissoras de pimenta biquinho com frutos de coloração salmão que possam ocupar um nicho do crescente mercado nacional de pimentas.

\section{MATERIAL E MÉTODOS}

Em março de 2009, um lote de pimenta biquinho vermelha cultivado na Embrapa Hortaliças apresentou grande variabilidade quanto ao porte das plantas, coloração e formato dos frutos. Com o objetivo de aumentar a frequência do alelo que confere a coloração salmão observada em alguns frutos, sementes foram extraídas daqueles frutos que apresentaram esta coloração. Estas sementes foram semeadas e as plântulas obtidas transplantadas para o campo, quando se obteve uma população de 500 plantas. A área de cultivo desta população foi dividida em 5 parcelas iguais, com o objetivo de realizar seleção massal estratificada para plantas com frutos de coloração salmão, além de outras características fenotípicas desejáveis, tais como, formato da copa, altura da planta e estado fitossanitário, avaliado por meio de uma estimativa visual da sanidade da planta. Critérios adicionais de seleção incluíram formato, cor e sabor dos frutos, assim como uma avaliação visual da produtividade por planta. Com base nestes critérios foram selecionadas 50 plantas. Esses 50 genótipos foram semeados individualmente e posteriormente transplantados para vasos e mantidos em casa de vegetação onde foram realizados dois ciclos de autofecundação. Após o segundo ciclo de autofecundação, 17 progênies (CNPH 35.093; CNPH 35.094, CNPH 35.095; CNPH 35.096; CNPH 35.097; CNPH 35.098; CNPH 35.099; CNPH 35.100; CNPH 35.101; CNPH 35.103; CNPH 35.104; CNPH 35.105; CNPH 35.106; CNPH 35.107; CNPH 35.108; CNPH 35.110 e CNPH 35.111) foram caracterizadas morfologicamente utilizando-se 11 descritores propostos pelo Bioversity International (IPGRI, 1995) e avaliadas agronomicamente em campo quanto à sua produção. Adicionalmente, determinou-se o teor de capsaicina utilizando-se cromatografia líquida de alta eficiência (HPLC, High Pressure Liquid Chromatography), de acordo com o método AOAC (Grypa, 2011). A condução das plantas em campo seguiu as orientações agronômicas descritas em Ribeiro et al. (2008).

Caracterização morfológica - Foi realizada em junho de 2012 em 17 progênies, com base em 11 descritores, sendo utilizados sete descritores de fruto: comprimento $(\mathrm{mm})$; largura $(\mathrm{mm})$; superfície (lisa ou áspera); massa de fruto fresco $(\mathrm{kg})$; comprimento do pedicelo $(\mathrm{mm})$; espessura da parede $(\mathrm{mm})$ e número de lóculos; três descritores de semente: cor, superfície (lisa ou áspera) e número de sementes por fruto; e um descritor de planta: pungência de frutos maduros. Para essa caracterização foram considerados os valores médios de cinco frutos maduros colhidos em cada progênie.

Adicionalmente foram coletadas informações sobre o comprimento do bico do fruto e aroma. O comprimento do bico foi medido a partir do ponto em que o fruto atingia $5 \mathrm{~mm}$ de largura, considerando os valores médios medidos com auxílio de paquímetro digital em cinco frutos maduros de cada progênie; quanto ao aroma, este foi determinado com teste sensorial realizado por um avaliador que classificou os frutos como tendo 
aroma nulo, médio ou forte, a partir da degustação de 5 frutos de cada progênie, já que aroma, além de cor e pungência têm sido usados como parâmetros de qualidade para a avaliação de hortaliças e frutas frescas (Oruna-Concha et al., 1998). As características morfológicas não foram analisadas estatisticamente.

Estimativa de produção - Foi estimada por meio de uma única colheita realizada em 10 de agosto de 2012, quando todas as progênies possuíam pelo menos uma planta com frutos maduros. Para cada progênie foram colhidos todos os frutos maduros presentes nas três plantas. A colheita foi realizada com auxílio de uma derriçadeira de café Stihl ${ }^{\odot}$ portátil, motorizada, com hastes duplas de 20 dedos. Os frutos derriçados foram recolhidos em uma lona plástica. Posteriormente, os frutos foram pesados em balança de precisão. Os dados foram analisados considerando-se o teste não paramétrico de Kruskal-Wallis, usado para amostras independentes e aleatórias, adotando-se um nível de significância $\alpha=1 \%$ (Zar, 1974) em razão de não ter sido adotado nenhum delineamento experimental.

Caracterização química - Foi realizada em fevereiro de 2013, através do método AOAC Official Method (Grypa, 2011) para determinação do teor de capsaicina entre 750 e 650.000 SHUs. A determinação dos capsaicinoides foi realizada somente em amostras de quatro progênies, que foram selecionadas devido às suas características morfológicas, aroma e estimativa de produção. Cinco frutos maduros de cada uma das quatro progênies foram cortados em pedaços de aproximadamente $0,3 \mathrm{~mm}$, e colocados em estufa, com ventilação forçada de ar, para secagem durante três dias à temperatura de aproximadamente $60^{\circ} \mathrm{C}$. As amostras secas foram trituradas em moinho e pesadas em alíquota de 12,5 g por progênie e colocadas em um frasco ao qual se adicionou 100 $\mathrm{mL}$ de etanol desnaturado. Os frascos foram acoplados a um condensador por refluxo e mantidos a $80^{\circ} \mathrm{C}$, durante cinco horas. Ao final deste período, o sobrenadante foi recolhido e mantido em geladeira a $4^{\circ} \mathrm{C}$, para posterior análise por cromatografia líquida de alta eficiência (HPLC, High Pressuree Liquid

\section{Chromatography).}

Após as quatro horas, as amostras foram filtradas através de uma membrana FG (fluoropore) em PTFE 0,45 $\mu \mathrm{m}$ de poro, hidrofóbica, com auxílio de uma seringa de $5 \mathrm{~mL}$, coletando $2 \mathrm{~mL}$ do material filtrado em vials para HPLC. As condições do HPLC (equipamento da marca Shimadzu ${ }^{\odot}$ ) foram formadas por uma fase móvel composta por acetonitrila $(40 \%)$ e água contendo $1 \%$ de ácido acético $(60 \%)$ que atravessa uma fase estacionária formada pela coluna C18 Phenomenex ${ }^{\odot}$ modelo Luna $5 \mathrm{u}$ C18(2) 100A, com tamanho de 150x4,6 $\mathrm{mm}$ a um fluxo de $1,5 \mathrm{~mL}$ por minuto, resultando em um tempo total de corrida de 30 minutos para cada amostra. Foram injetados $20 \mu \mathrm{L}$ de cada amostra em duplicatas, que percorreram a fase estacionária juntamente com a fase móvel. Os picos de capsaicinoides foram obtidos a partir de um detector do tipo fotodiodo (PDA, Photodiode Array Detector) UV-vis (ultravioleta visível), utilizando-se como referência o comprimento de onda de $280 \mathrm{~nm}$. A identificação dos capsaicinoides foi baseada na comparação do tempo de retenção dos picos relativos aos capsaicinoides encontrados em amostras de padrões comerciais de capsaicina e dihidrocapsaicina (marca Extrasynthese $\left.^{(}\right)$. A quantificação da capsaicína foi feita a partir da comparação da área do pico obtida da injeção de dois padrões de capsaicina (Padrão A na concentração de $0,015 \mathrm{mg} / \mathrm{mL}$ e Padrão $\mathrm{B}$ a $0,15 \mathrm{mg} / \mathrm{mL}$ ) com as áreas obtidas nos picos correspondentes à capsaicina da injeção, a cada seis amostras injetadas, com base na fórmula a seguir: $\mathrm{C}=$ (Pc/Ps) x (Cs/Wt) x (200/0,89) x 16100, onde $\mathrm{C}=$ quantidade de capsaicina em $\mathrm{SHU} ; \mathrm{Pc}=$ área do pico relativo à capsaicina; $\mathrm{Os}=$ área do pico relativo ao padrão de capsaicina; $\mathrm{Cs}=$ concentração do padrão de capsaicina; $\mathrm{Wt}=$ massa $(\mathrm{g})$ da amostra teste.

Os resultados não foram analisados estatisticamente.

\section{RESULTADOS E DISCUSSÃO}

Caracterização morfológica - $\mathrm{Na}$ caracterização morfológica identificou-se a existência de variabilidade entre as progênies, sendo observadas variações para todas as características de planta e fruto estudadas.

A largura média do fruto variou entre 12,1 mm para CNPH 35.093 e 16,6 mm para CNPH 35.103. O comprimento médio do bico do fruto variou entre 1,5 mm para CNPH 35.097 e 6,3 mm para CNPH 35.103 e 35.112. Com relação ao comprimento médio do fruto houve variação entre $19,8 \mathrm{~mm}$, para $\mathrm{CNPH}$ 35.095, CNPH 35.098 e CNPH 35.100, e $28,5 \mathrm{~mm}$, para CNPH 35.103 (Tabela 1). Segundo Rêgo (2001), determinadas características morfológicas como maior comprimento dos frutos têm grande importância em função da sua alta correlação com outros caracteres de interesse como a produtividade. As progênies CNPH 35.094, CNPH 35.103 e CNPH 35.112, apesar de possuírem características morfológicas diferenciadas entre si, destacaram-se por apresentar frutos muito uniformes e vistosos.

Todas as progênies estudadas apresentaram superfície lisa do fruto. A espessura média da parede do fruto variou de 1,6 a 2,3 mm, sendo que oito progênies apresentaram espessura média entre 2,0 e 2,1 mm. As progênies CNPH 35.095, CNPH 35.105, CNPH 35.107 e CNPH 35.112 apresentaram maiores espessuras. Progênies com maior espessura da parede do fruto são interessantes comercialmente em função do consumo da pimenta biquinho ocorrer preferencialmente na forma de conservas. $\mathrm{O}$ número médio de lóculos variando entre três e quatro foi o mais frequente.

A determinação das características do fruto é muito importante para o cultivo de pimentas, visto que o mercado consumidor determina a forma de consumo e a sua aceitação, ou seja, diferentes nichos de mercado demandam por pimentas com características específicas de fruto (Dagnoko et al., 2013; Ribeiro et al., 2008).

O comprimento médio do pedicelo variou entre $17,7 \mathrm{~mm}$ para $\mathrm{CNPH}$ 35.104 e 26,1 mm para CNPH 35.112.

Todas as sementes dos frutos caracterizados apresentaram cor amarela-pálida e superfície lisa. O número médio de sementes por fruto variou de $82(\mathrm{CNPH}$ $35.093)$ a 213 (CNPH 35.099). 
Tabela 1. Caracterização de 17 progênies de pimenta biquinho salmão para descritores de frutos, semente e planta (valores médios para cinco frutos) (characterization of 17 lines of salmon-colored "biquinho"-type pepper using fruit, seed and plant descriptors). Brasília, Embrapa Hortaliças, 2012.

\begin{tabular}{|c|c|c|c|c|c|c|c|c|c|}
\hline $\begin{array}{l}\text { Progênie } \\
\text { (CNPH } \\
\text { número) }\end{array}$ & $\begin{array}{c}\text { Comprimento } \\
\text { (mm) }\end{array}$ & $\begin{array}{l}\text { Largura } \\
(\mathbf{m m})\end{array}$ & $\begin{array}{l}\text { Comprimento } \\
\text { do bico }(\mathrm{mm})\end{array}$ & $\begin{array}{l}\mathrm{N}^{\circ} \mathrm{de} \\
\text { lóculos }\end{array}$ & $\begin{array}{l}\text { Comprimento } \\
\text { do pedicelo } \\
(\mathbf{m m})\end{array}$ & $\begin{array}{l}\text { Espessura } \\
\text { da parede } \\
(\mathrm{mm})\end{array}$ & Aroma & $\begin{array}{c}\text { Massa/ } \\
\text { planta } \\
(\mathbf{k g})\end{array}$ & Superfície \\
\hline 35.093 & 20,1 & 12,1 & 3,6 & 2,2 & 23,2 & 2,0 & Forte & 0,6 & Lisa \\
\hline 35.094 & 22,3 & 14,6 & 5,6 & 2,6 & 20,2 & 1,8 & Fraco & 1,7 & Lisa \\
\hline 35.095 & 19,8 & 14,3 & 3,0 & 2,6 & 20,5 & 2,3 & Médio & 1,1 & Lisa \\
\hline 35.096 & 25,4 & 15,2 & 3,0 & 3,0 & 20,3 & 1,6 & Médio & 1,1 & Lisa \\
\hline 35.097 & 22,1 & 13,8 & 1,5 & 3,2 & 22,5 & 2,0 & Médio & 1,5 & Lisa \\
\hline 35.098 & 19,8 & 15,3 & 3,1 & 2,8 & 21,6 & 1,8 & Fraco & 1,4 & Lisa \\
\hline 35.099 & 20,4 & 16,1 & 2,1 & 3,8 & 21,8 & 1,9 & Fraco & 1,7 & Lisa \\
\hline 35.100 & 19,8 & 15,0 & 2,8 & 3,4 & 20,3 & 1,7 & Médio & 0,9 & Lisa \\
\hline 35.101 & 21,8 & 15,0 & 3,6 & 2,8 & 17,9 & 2,1 & Fraco & 1,2 & Lisa \\
\hline 35.103 & 28,5 & 16,6 & 6,3 & 2,8 & 22,7 & 2,1 & Forte & 1,3 & Lisa \\
\hline 35.104 & 23,5 & 15,9 & 3,9 & 3,2 & 17,7 & 2,1 & Fraco & 1,2 & Lisa \\
\hline 35.105 & 22,0 & 15,4 & 4,8 & 2,2 & 19,6 & 2,3 & Médio & 0,9 & Lisa \\
\hline 35.106 & 26,1 & 15,1 & 5,8 & 2,4 & 26,0 & 2,0 & Médio & 0,4 & Lisa \\
\hline 35.107 & 22,0 & 14,5 & 4,5 & 2,4 & 20,5 & 2,2 & Fraco & 0,7 & Lisa \\
\hline 35.108 & 22,6 & 15,2 & 4,9 & 2,8 & 23,5 & 2,0 & Fraco & 0,1 & Lisa \\
\hline 35.111 & 20,2 & 15,9 & 3,5 & 3,0 & 25,7 & 2,1 & Médio & 0,0 & Lisa \\
\hline 35.112 & 22,4 & 14,4 & 6,3 & 2,8 & 26,1 & 2,2 & Médio & 0,1 & Lisa \\
\hline
\end{tabular}

As progênies avaliadas possuem aroma característico: dez delas apresentaram aroma forte ou médio. É interessante mencionar que Pino et al. (2007) concluíram que pimentas com frutos de cores laranja e marrom possuem melhor composição química relacionada ao aroma do que variedades de frutos vermelhos. Pinto et al. (2010) também identificaram a presença de compostos voláteis que conferem características desejáveis de aroma em linhagens de pimenta biquinho. Entre as progênies que apresentaram forte aroma, duas se destacaram, CNPH 35.093 e CNPH 35.103, constituindo assim, progênies promissoras. Koeda et al. (2014) concluíram que existem poucas cultivares de $C$. chinense que sejam não pungentes e ao mesmo tempo altamente aromáticas, sendo a união dessas duas características, um alvo a ser considerado por programas de melhoramento.

Estimativa de produção - Foi detectada diferença significativa na produção das progênies, sendo que $\mathrm{CNPH}$ 35.094 e CNPH 35.099 apresentaram as maiores médias de produção, 1,73 e 1,68 $\mathrm{kg} /$ planta, respectivamente (Tabela 2). Outras progênies também se destacaram com boas médias de produção: CNPH 35.097 (1,46 kg/planta), CNPH 35.098 $(1,40 \mathrm{~kg} /$ planta $)$ e CNPH 35.103 (1,32 $\mathrm{kg} /$ planta). Apesar de não existirem cultivares de pimenta biquinho com frutos de coloração salmão no mercado, cultivares com coloração vermelha, como BRS Moema, comumente produzem 20 t/ha (EMBRAPA, 2012). No espaçamento de 1,2x0,6 m, como utilizado na produção de pimenta biquinho, a média das produções observadas por planta para as três progênies acima, equivaleria a mais de $19 \mathrm{t} / \mathrm{ha}$.

Caracterização química - As progênies selecionadas com base nas características morfológicas, aroma e estimativa de produção, foram CNPH 35.093, CNPH 35.094, CNPH 35.099 e CNPH 35.103. Os teores médios de capsaicina encontrados nessas progênies foram muito baixos: 116; 185; 272 e 95 SHUs, respectivamente. Esses valores estão de acordo com a literatura, que indica que apesar de presente, estes teores não determinam pungência ao paladar humano (Ribeiro et. al., 2008). Todavia, Domenico et al. (2012) não detectaram capsaicina para o acesso de pimenta biquinho analisado (IAC 1643). Além disso, é importante ressaltar que a concentração de capsaicina nos frutos de pimenta pode ser afetada por fatores genéticos, fisiológicos, ambientais e inclusive pelos tratos culturais (Bosland \& Votava, 2000; Ribeiro et al., 2008). A influência desses fatores, além da diferença no método adotado e nas condições de extração, pode explicar as variações observadas entre Domenico et al. (2012) e este trabalho.

Neste trabalho foi possível observar variabilidade genética em uma população de pimenta biquinho, em termos de cor do fruto, superfície e número de sementes, comprimento, largura, massa de fruto fresco, comprimento do pedicelo, espessura da parede do fruto, número de lóculos e pungência de frutos maduros. Essa variabilidade pode ser utilizada em programas de melhoramento explorando-se métodos como a seleção massal. Uma progênie (CNPH 35.103) foi considerada promissora, 
Tabela 2. Média da produção por planta para cada uma das 17 progênies de pimenta biquinho salmão analisadas. P-valor equivalente a 0,00367118 (grau de liberdade 16 e qui-quadrado equivalente a 35,25133479) \{average production per plant for the 17 lines of salmon-colored "biquinho"- type pepper. P-value equivalent to 0.00367118 (degrees of freedom 16 and $\mathrm{chi}^{2}$ equivalent to 35.25133479)\}. Brasília, Embrapa Hortaliças, 2012.

\begin{tabular}{lccc}
\hline $\begin{array}{l}\text { Progênie } \\
\text { (CNPH número) }\end{array}$ & Média/planta (kg) & $\begin{array}{c}\text { Progênie (CNPH } \\
\text { número) }\end{array}$ & $\begin{array}{c}\text { Média/planta } \\
(\mathbf{k g})\end{array}$ \\
\hline 35.093 & 0,55 & 35.103 & 1,32 \\
35.094 & 1,73 & 35.104 & 1,22 \\
35.095 & 1,14 & 35.105 & 0,87 \\
35.096 & 1,08 & 35.106 & 0,38 \\
35.097 & 1,46 & 35.107 & 0,71 \\
35.098 & 1,40 & 35.108 & 0,07 \\
35.099 & 1,68 & 35.110 & 0,04 \\
35.100 & 0,85 & 35.111 & 0,10 \\
35.101 & 1,18 & & \\
\hline
\end{tabular}

pois apresentou boa produção, pungência praticamente nula, forte aroma e parede do fruto mais espessa quando comparada às demais, demonstrando que pode dar origem a uma cultivar de pimenta biquinho com frutos de cor salmão. As progênies CNPH 35.094 e 35.099 se destacaram entre as demais por apresentarem maior produção de frutos ( $\mathrm{kg} /$ planta) e teores de capsaicina próximos a zero, constituindo-se em genótipos com alto potencial para uso comercial.

\section{AGRADECIMENTOS}

A primeira autora agradece ao professor José Ricardo Peixoto, da Faculdade de Agronomia e Medicina Veterinária da Universidade de Brasília, pela sua contribuição ao trabalho de conclusão de curso que serviu como base para o desenvolvimento desse artigo.

\section{REFERÊNCIAS}

ALVARES RC; REIS EF; PINTO JFN. 2012. Genetic divergence in pepper genotypes from southwest Goiás. Ciência e Agrotecnologia 36: 498-506.

BOSLAND PW; BARAL JB. 2007. 'Bhut Jolokia': the world's hottest known chilly pepper is a putative naturally occurring interspecific hybrid. HortScience 42: 222-224.

BOSLAND PW; VOTAVA EJ. 2000. Peppers: vegetable and spice Capsicum. Berkeley: Ten Speed Press. 240p.

CARVALHO SIC; BIANCHETTI L;
BUSTAMANTE PG; SILVA DB. 2003. Catálogo de germoplasma de pimentas e pimentões (Capsicum spp.) da Embrapa Hortaliças. Brasília: Embrapa Hortaliças, (Embrapa Hortaliças. Documentos, 49), 49 p.

CARVALHO SIC; BIANCHETTI LB; REIFSCHNEIDER FJB. 2009. Registro e proteção de cultivares pelo setor público: a experiência do programa de melhoramento de Capsicum da Embrapa Hortaliças. Horticultura Brasileira 27: 135-138.

CARVALHO SIC; BIANCHETTI LB; RIBEIRO CSC; LOPES CA. 2008. Pimentas do gênero Capsicum no Brasil. Brasília: Embrapa Hortaliças, 27p.

DAGNOKO S; YARO-DIARISSO N; SANOGO PN; ADETULA, O; DOLO-NATOUMÉ A; GAMBY-TOURÉ K; TRAORÁ-THÉRA 1; KATILÉ S; DIALLO-BA D. 2013. Overview of pepper (Capsicum spp.) breeding in West Africa. African Journal of Agricultural Research 8: 1108-1114.

DE WITT D; BOSLAND PW. 2009. The complete chilli pepper book - A gardener's guide to choosing, growing, preserving and cooking. London: Timber Press. 336 p.

DOMENICO CI; COUTINHO JP; GODOY HT; MELO AMT. 2012. Caracterização agronômica e pungência em pimenta de cheiro. Horticultura Brasileira, 30: 466-472.

EMBRAPA. Agência de Informação Embrapa. 2012. Pré-produção, Características, Cultivares, Capsicum chinense. Disponível em:<http://www.agencia. cnptia.embrapa.br/gestor/pimenta/arvore/ CONT000gn0frh1202wx5ok0liq1mqt5bf5ht. html $>$. Acessado em 12 de março, 2015.

FAO. Food and Agriculture Organization of the United Nations. 2012. FAOSTAT. Disponível em: <http://faostat.fao.org/>. Acessado em 21 de março, 2013.

FARIA PN; LAIA GA; CARDOS KA; FINGER FL; CECON PR. 2013. Estudo da variabilidade genética de amostras de pimenta (Capsicum chinense) existentes num banco de germoplasma: um caso de estudo. Revista de
Ciências Agrárias 36: 17-22.

GARRUTI DS; PINTO NOF; ALVES VCC; PENHAMFAA; TOBARUELAEC; ARAÚJO IMS. 2013. Volatile profile and sensory quality of new varieties of Capsicum chinense. Ciência e Tecnologia de Alimentos 33: 102108.

GRYPA R. 2011. Spices and other condiments. In: HORWITZ W; LATIMER GW. (eds). Official methods of analysis of association of official analytical chemists international. Gaithersburg: AOAC. p.14-15.

IPGRI. 1995. Descritores para Capsicum (Capsicum spp.). Roma: IPGRI, 51p.

JARRET RL; BERKE T. 2008. Variation for fruit morphological characteristics in a Capsicum chinense germplasm collection. HortScience 43: 1694-1697.

KOEDA S; KOSUKE S; KENICHI T; YOSHIYUKI T; RIHITO T; MUNETAKA H; MOTOAKI D; TETSUYA N; AKIRA K. 2014. Analysis of non-pungency, aroma, and origin of a Capsicum chinense cultivar from a Caribbean Island. Journal of the Japanese Society for Horticultural Science. doi: 10.2503/jjshs1.CH-105 (no prelo).

KOSUGE S; FURUTA M. 1970. Studies on the pungent principle of Capsicum. Part XIV: chemical constitution of the pungent principle. Journal of Agricultural and Biological Chemistry 34: 248-256.

MATERSKA M; PERUCKA I. 2005. Antioxidant activity of the main phenolic compounds isolated from hot pepper fruit (Capsicum annuum). Journal of Agricultural and Food Chemistry 53: 1750-1756.

MOSCONE EA; SCALDAFERRO MA; GRABIELE M; CECCHINI NM; GARCÍA YS; JARRET R; DAVIÑA JR; DUCASSE DA; BARBOZA GE; EHRENDORFER F. 2007. The evolution of chilli peppers (Capsicum - Solanaceae): a cytogenetic perspective. Acta Horticulturae 745: 137-169.

MOSES M; UMAHARAN P. 2012 Genetic structure and phylogenetic relationships of Capsicum chinense. Journal of the American Society for Horticultural Science 137: 250262.

MOURA MCCL; GONÇALVES LSA; SUDRÉ CP; RODRIGUES R; AMARAL JÚNIOR AT; PEREIRA TNS. 2010. Algoritmo de Gower na estimativa da divergência genética em germoplasma de pimenta. Horticultura Brasileira 28: 155-161.

OR UNA - C ONCH A M J ; LÓ P E Z HERNÁNDEZ J; SIMAL-LOZANO J; SIMAL-GÁNDARA J; GONZÁLEZCASTRO MJ; CRUZ-GARCÍA C. 1998. Determination of volatile components in fresh, frozen and freezed-dried "Padrón-type" peppers by GC-MS using dynamic headspace sampling and microwave desorption. Journal of Chromatographic Science 36: 583-88.

PINO J; GONZÁLEZ M; CEBALLOS L; CENTURIÓN-YAH AR; TRUJILLOAGUIRRE J; LATOURNERIE-MORENO L; SAURI-DUCH E. 2007. Characterization of total capsaicinoids, color and volatile compounds of habanero chilli pepper (Capsicum chinense) cultivars in Yucatan. 
Food Chemistry 104: 1682-1686.

PINTO NOF; GOMES BL; SILVA THS; PENHA

MFA; ALVEZ VCC; GARRUTI DS. 2010.

Análise de compostos do aroma da pimenta

biquinho laranja (C. chinense) por CG-

EM e CG-olfatometria. In: SIMPÓSIO

BRASILEIRO DE CROMATOGRAFIA E

TÉCNICAS AFINS, 4. Resumos... São Carlos:
Instituto Internacional de Cromatografia (CD-ROM).

RÊGO ER. 2001. Diversidade, herança e capacidade combinatória em pimenta (Capsicum baccatum). Viçosa: UFV. 117p (Tese doutorado).

RIBEIRO CSC; LOPES CA; CARVALHO SIC; HENZ GP; REIFSCHNEIDER FJB. 2008 (eds). Pimentas Capsicum. Brasília: Embrapa Hortaliças, 200p.

SOUZA SAM; MARTINS KC; PEREIRA

TNS. 2011. Polimorfismo cromossômico em Capsicum chinense. Ciência Rural 41: 1777-1783.

ZAR JH. Biostatistical analysis. 1974. Upper Saddle River: Prentice-Hall, 620p. 